\title{
EL COMPONENTE GRAMATICAL EN LAS CREENCIAS LINGÜÍSTICAS: DIFERENCIA Y JERARQUÍA, CORRECCIÓN Y VARIACIÓN
}

\author{
THE GRAMMATICAL COMPONENT IN LINGUISTIC BELIEFS: \\ DIFFERENCE AND HIERARCHY, CORRECTNESS AND VARIATION
}

\author{
CARMen Fernández Juncal \\ Universidad de Salamanca \\ cjuncal@usal.es
}

Enviado: $11 / 08 / 2017$

Aceptado: 08/05/2018

\section{Resumen}

Este artículo indaga en la presencia del componente morfosintáctico en las creencias de los hablantes frente a la presencia mayoritaria de los niveles fonético y léxico, que, como se ha puesto de manifiesto tradicionalmente, han acaparado el foco de los hablantes en la percepción y descripción lingüísticas. A partir de una serie de encuestas en un territorio perteneciente a la modalidad centro-peninsular del español y a la vista de los resultados cuantitativos, podemos confirmar ese papel subsidiario de la gramática. No obstante, la gramática adquiere una posición protagonista cuando las cuestiones planteadas a los hablantes incluyen el concepto de corrección, ámbito de carácter dicotómico en el que los aspectos gramaticales ofrecen más nitidez frente a la diversidad y ausencia de norma de referencia clara de la fonética y el léxico. En estos niveles la variación es evidente para los encuestados pero sin establecer una jerarquía entre las variantes coexistentes.

PALABRAS CLAVE: Creencias, conciencia morfosintáctica, modalidad centro-peninsular, corrección gramatical.

\begin{abstract}
This article delves into the presence of the morphosyntactic component in the beliefs of the speakers. As shown traditionally, it is two other levels, phonetic and lexical, which almost monopolize the speakers' attention in the linguistic perception and description. From a series of surveys in an area belonging to the Iberian Peninsula Central variety and in view of our quantitative results, we can confirm that subsidiary role of grammar. Nevertheless, grammar features become central when we move the attention to the dichotomous concept of correctness. In this context the morphosyntactic aspects offer sharper contrasts against the diversity and lack of clear criterion of the other levels studied. Variation in Phonetics and Lexicon is evident for the survey respondents but without establishing a hierarchy between the coexisting variants.

KEYWORDS: Beliefs, syntactic awareness, center-peninsular variety, grammatical correctness.
\end{abstract}

Para citar este artículo / To cite this article: Para citar este artículo / To cite this article: Fernández Juncal, Carmen (2018). El componente gramatical en las creencias lingüísticas: diferencia y jerarquía, corrección y variación. ELUA, 32: 111-129. doi: 10.14198/ELUA2018.32.5

Enlace / Link: http://dx.doi.org/10.14198/ELUA2018.32.5 


\section{MARCO TEÓRICO}

Siguiendo a Rojas (2012: 140), una actitud es "la valoración positiva o negativa que un individuo o grupo muestra hacia un rasgo lingüístico, una variedad lingüística completa o los usuarios de dicho rasgo o variedad" mientras que las creencias, entendidas como parte de las habilidades cognitivas de los individuos, son parte de ideologías o culturas lingüísticas enmarcadas en contextos socioculturales específicos. Las actitudes se manifiestan ordenadas en polos opuestos de valoración, pero, a efectos de la presente investigación, conviene destacar los señalados por Coseriu (1993) entre juicios lingüísticos (corrección-incorrección) y los juicios no lingüísticos (inteligencia, alegría, etc.). Estos últimos son mucho más numerosos en cuanto reflejan una actitud hacia los hablantes más que hacia su lengua. Por otra parte, se añade otro factor a la evaluación de una variedad, la teoría de la acción razonada ${ }^{1}$, que defiende que las creencias basadas en experiencias directas con un objeto predicen mejor las conductas basadas en esas mismas creencias ${ }^{2}$.

La importancia de las creencias explícitas de los hablantes ha sido puesta de manifiesto desde los primeros pasos de la Sociolingüística (Agheyisi y Fishman 1970, Cooper y Fishman 1974 y Giles y Bouchard Ryan 1982), también hispana (los pioneros Alvar 1977 y Lope Blanch 1986 y posteriormente, Borrego Nieto 1981 y 1992, Moreno y Moreno 2002, Cestero y Paredes 2013, 2015a y 2015b, Chiquito y Quesada 2014 y Buzón García et alii 2017, entre otros), pero no han constituido nunca el centro de las investigaciones de la disciplina aunque resultan determinantes en la configuración de los patrones de prestigio, el movimiento y dirección de los cambios lingüísticos o la supervivencia de usos tradicionales (López Morales 2004 [1989]). Por otra parte, hay que recordar también que las convicciones lingüísticas en general no tienen que basarse necesariamente en realidades constatables (Moreno Fernández 2000) y que en ocasiones la labor del investigador consiste en poner en evidencia las contradicciones entre la actuación lingüística y las manifestaciones explícitas de los hablantes (Fernández Juncal 2005 y en prensa).

Si repasamos la escasa bibliografía acerca de las creencias ${ }^{3}$, observamos que se definen en torno a diferentes conceptos: el de juicio ${ }^{4}$, el de conocimiento ${ }^{5}$ pero también relacionadas con el de conciencia (López Morales 2004 [1989]), el de percepción (Caravedo 2014) o el de ideología o cultura lingüísticas (Rojas 2012).

\footnotetext{
1 En el original theory of reasoned action. Cfr. Ajzen y Fishbein (1980).

2 Esa teoría a veces choca con la de los prototipos lingüísticos, que López García (1988) define como una necesidad cognitiva que se manifiesta en una conciencia dialectal y que asigna prestigio a las variedades más cercanas al prototipo y menos a las más alejadas de él. Por otra parte, Moreno Fernández (2001) recoge las diferentes teorías que intentan explicar los modelos de tipificación de las variedades lingüísticas.

3 "No se trata de un tema de estudio priorizado en el marco de la Sociolingüística, por lo que son pocas las investigaciones llevadas a cabo y, por tanto, escasos los conocimientos que tenemos sobre su funcionamiento y las repercusiones" (Cestero y Paredes, 2013:1)

4 "Son juicios de probabilidad de una persona respecto de un determinado aspecto de su mundo, su identidad y su contexto; o sea, el individuo asocia el objeto de creencia a varios atributos para formar o aprender creencias sobre sí mismo, otras personas, instituciones, acciones, etc.” (Espinosa Taset, 2009: 77).

5 Palomino Hernández (2013: 7) afirma que se trata de "las nociones que el individuo posee sobre el conocimiento de un objeto y que considera ciertas, por lo que forman la base de sus opiniones y de su actitud hacia ese objeto". Por su parte, Caravedo (2014: 47) las considera "tanto el conocimiento adquirido de una lengua, como el proceso que se pone en juego para conocerla y utilizarla”.
} 
En cualquier caso, no podemos pensar que la percepción de la lengua por parte de sus usuarios es un instrumento infalible para los lingüistas. Hay que tener en cuenta, en primer lugar, que las manifestaciones metalingüísticas reflejan muchas veces en realidad actitudes hacia los usuarios más que hacia los propios elementos lingüísticos sometidos a juicio (Milroy 2001, Edwards 2011). En segundo lugar, en muchas ocasiones tratamos con sujetos que tienen un contacto muy restringido con la variedad estándar del español y con otras variedades regionales. Además, no tenemos la certeza de que sus respuestas no estén motivadas más por estereotipos (positivos o negativos) o por modas que por verdaderas convicciones, producto de su experiencia. Recordemos de nuevo que las creencias de los hablantes están inmersas en el ámbito más global de las actitudes sociolingüísticas y todas ellas se vinculan a aspectos de tipo extralingüístico. Esto último no ha de extrañar habida cuenta de que la lengua es, para los hablantes, una realidad extracognitiva, externa al individuo, que existe más allá del uso de sus hablantes y que posee cualidades propias de los humanos y de otros objetos reales (Moreno Fernández 2007). Por esta razón las creencias acaban por tener influencia en la actuación lingüística, como bien resume López Morales (2004 [1989]: 291):

Aunque no todas las creencias producen actitudes (piénsese, por ejemplo, en las etimologías populares) en su mayoría conllevan una toma de posición: si se cree que el fenómeno $\mathrm{x}$ es rural, es decir, lleva signos de rusticidad, inelegancia, etc., suele producirse una actitud negativa hacia él, se suele rechazar. Que tal rechazo afecta a la actuación lingüística del hablante es un hecho, sobre todo cuando produce estilos cuidadosos en los que participa muy activamente su conciencia lingüística.

En lo que concierne a la conciencia de los hablantes respecto a los diferentes niveles lingüísticos, los trabajos previos presentan diversidad de opiniones. Así, en muchos de ellos es el nivel léxico el que presenta más alusiones. Es lo que ocurre en la investigación de Ellis (1969: 111):

A majority of those present at the Bradford meeting claimed to be able to tell if a speaker was a native of Bradford but could not always explain why. Most often the difference came in the use of lexical items although phonology was clearly important.

Lo ratifica Mase (1964: 363) cuando destaca que los entrevistados para su investigación hacen 102 referencias al léxico y 94 a la fonética, seguidos a más distancia de la gramática. Cazacu (1968: 1462) entiende que el proceso de interacción entre dialecto y lengua estándar no es igual en los planos fonológico y morfológico, más cerrados, que en el léxico, de carácter más abierto y con más posibilidades, por lo tanto, de ser motivo de cambio y de penetración de la lengua estándar en los dialectos. Este autor comprueba cómo el sistema léxico de un dialecto (rumano en este caso) permite la adopción de vocablos importados, pero los adapta a los hábitos fonéticos locales. Por consiguiente, la fonética y la gramática son, para el lingüista rumano, los elementos más conservadores de la modalidad regional dentro de una forma intermedia de comunicación llamada interdialecto, situada entre el aspecto estándar y el aspecto dialectal de la lengua. Por otra parte, la investigación de López Morales (realizada en 1978 y recogida en 2004 [1989]) en San Juan de Puerto Rico también señala que la conciencia de los interrogados, además de estar en correlación directa con su nivel socioeconómico, es más perceptiva de los fenómenos léxicos que de los fonéticos y 
que ambos se sitúan muy por encima de los sintácticos en el grado de aparición, sobre todo en las clases populares, menos sensibles a los elementos estructurales que a los pertenecientes a otros niveles de la lengua.

Sin embargo, hay referencias que defienden el valor de lo fónico a la hora de establecer juicios lingüísticos. Anglejan y Tucker (1973) refrendan su mayor presencia dentro de los fenómenos citados en su investigación en Quebec. Es la misma postura que defiende Boyer (1991: 43),

Du point de vue social, la forme phonique, qui constitue ce que nous percevons en premier d'un locuteur, fait l'objet de jugements nombreux et semble bien être, comme le remarque Françoise Gadet "la dimensión la plus classante de la langue".

Así, es muy habitual que el hablante perciba diferencias en el acento, la entonación o el deje, tres términos que resumen las respuestas mayoritarias en este ámbito y que se caracterizan en el habla especializada por su imprecisión y dificultad de análisis. En este sentido Milroy (1982: 141) destaca los escollos para la observación de estas percepciones de carácter fonético-fonológico, muy claras para los hablantes pero apenas definibles desde el punto de vista de la lingüística:

The notion of "accent" appears to be psycho-social rather than strictly linguistic. Thus, although accents clearly have a psycho-social reality in the sense that speakers show an awareness of their importance as markers of various aspects of social identity, and react correspondingly strongly to them, it is not possible to produce strict "phonological" definitions of accents any more than "dialects" can be seen as clear linguistic entities.

El peso del acento, como marca fónica del hablante, es decisivo en otras lenguas, como el inglés, donde tiene funciones de indicador social y generador de ideas y actitudes, hasta el punto de que, como señala Trudgill (1983a), la variación fonética se observa como una desviación de la norma y es descrita como un mal uso lingüístico ${ }^{6}$. Son los valores afectivos y grupales, la solidaridad frente al poder, los que contrarrestan esta ideología reprobadora y permiten el mantenimiento de los dialectos y acentos no estándares (Haenni 1999). Trudgill (1983b) explica esta situación a través de una doble hipótesis: por un lado, la del valor inherente, que defiende la adjudicación de rasgos positivos de atractivo a determinadas variedades, que adquieren así prestigio dentro de la comunidad lingüística ${ }^{7}$. Por otra parte, presenta la hipótesis de la norma impuesta, que sostiene que la evaluación de las variedades no se fundamenta tanto en cuestiones intrínsecas (de hecho, no hay razón alguna que haga más grata o atractiva una variedad u otra) sino por las diferentes presiones de tipo social y cultural que se ejercen en cada comunidad. Así, la relación entre la variación fonética y la distribución social está acreditada desde hace tiempo (Wells 1982), de manera que, a medida que descendemos en el espectro social, mayor alternancia de acentos se detecta.

6 Estos juicios tienen consecuencias sociales inmediatas afectando al ascenso y la promoción sociales o incluso a los resultados de un juicio o a un diagnóstico médico (Honey 1989). Hansen (2007) emplea la palabra estigmatización para describir la situación.

7 Así, la Received Pronunciation se coloca en la cúspide del prestigio asociado a valores como la belleza (Trudgill 1983b). 
Por último y en lo que se refiere a la preeminencia del componente fónico, quizá merezca consideración, aunque excede el ámbito de este estudio, el hecho de que la competencia fónica y fonológica, como parte de las habilidades cognitivas del ser humano, parece ser la primera en desarrollarse entre los aprendices de hablante y, con ella, la conciencia de este nivel $^{8}$. Como bien saben los educadores tanto de primera como de segunda lengua, se trata de un componente vital dentro de la conciencia lingüística (Cots et al. 2007) para tareas como el aprendizaje de las destrezas lectoescritoras (Moats 2000 y Ehri et al. 2001).

Hay una ausencia completa de investigaciones que pudieran considerar el nivel morfosintáctico como elemento muy presente en la conciencia de los hablantes, quizás porque, como afirma López Morales (2004 [1989]: 214), "la sintaxis apenas participa de las decisiones $[\ldots]$, lo que no es de sorprender, pues, en general, ofrece indicios complicados y no muy fácilmente verbalizables". En esta misma idea de complejidad de la gramática, entendida como "la construcción e interpretación de los enunciados", incide Martí Sánchez (2004: 4):

No es casualidad, por tanto, que se sostenga que la gramática es el medio más evolucionado con el que cuentan las lenguas para la comunicación (Givón 2001: 11-13), gracias al cual es posible "formar expresiones complejas para transportar significados complejos" (Dik 1997: 8); justamente, uno de los rasgos del pensamiento abstracto, última etapa de la maduración intelectual. No es casualidad tampoco que muchos errores de los hablantes inmaduros se encuentren en el manejo e interpretación de determinadas construcciones y otras herramientas gramaticales dotadas de una notable dosis de abstracción.

Este papel secundario de los rasgos morfosintácticos respecto a los fonéticos o los léxicos no excluye el interés de determinar su presencia, el grado de aparición de fenómenos con diferentes perfiles en la percepción de los usuarios de la lengua.

\section{METODOLOGÍA DE LA INVESTIGACIÓN}

Los datos que presentamos a continuación son los resultados de una encuesta llevada a cabo en el oriente de Cantabria, desde el río Pas hasta los límites con el País Vasco, a lo largo de la segunda mitad del año 2016. Se trata de la reproducción prácticamente idéntica de otra llevada a cabo en 1996, por lo tanto, con 20 años de diferencia, en el mismo territorio y con el objetivo de determinar las creencias de nuestros informantes acerca de diversas cuestiones de tipo sociolingüístico, como complemento para un estudio variacionista sobre dos fenómenos de carácter dialectal (Fernández Juncal 2000) ${ }^{9}$.

El cuestionario, basado fundamentalmente en el empleado por Borrego Nieto (1981), está formado por 32 preguntas, que indagan en torno a cuatro puntos fundamentales: la percepción de la norma o normas (lo que incluye los modelos de autoridad y los patrones de corrección de la comunidad y la presencia en ellos de los medios de comunicación social), la valoración del habla de la zona, la opinión sobre la situación sociolingüística de la comunidad y la consideración de las hablas limítrofes.

8 Según López-Ornat (1992), la gramaticalización solo comienza con las emisiones de dos palabras.

9 Se empleó entonces un cuestionario de tipo abierto en un colectivo de 84 informantes, residentes en 52 municipios diferentes con distribución normalizada por sexos, edades y nivel sociocultural. 
En 13 de las entradas empleadas se solicitaba explícitamente la concreción de los elementos lingüísticos que conducían a la valoración de la variedad en cuestión. Se trata de las siguientes preguntas:

- Sobre norma lingüística: “¿Puede ponerme algún ejemplo de algo que se diga mal en Cantabria?”, “¿Puede poner ejemplos de habla demasiado “fina”?”10, “Le han corregido a usted alguna vez por su forma de hablar?”, “¿Qué diferencia la forma de hablar de un locutor de radio o televisión y la de una persona de la calle?”, “¿En qué distinguiría a alguien que está leyendo de alguien que habla sin apoyo de un texto?".

- Sobre hablas limítrofes: “¿Distinguiría a una persona del País Vasco/ Castilla / Asturias por su forma de hablar? ¿En qué los distinguiría?”.

- Sobre variación sociolingüística interna: “¿Quiénes hablan mejor, los hombres o las mujeres?, ¿Los jóvenes o los mayores? ¿En los pueblos o en la ciudad?, ¿En la costa o en el interior? ¿Por qué'?”.

Estas preguntas provocaban manifestaciones que incluían algún ejemplo o explicación metalingüística. A partir de ellas, podemos establecer una clasificación de acuerdo con el nivel lingüístico al que estuvieran asociadas: ejemplificación en el nivel fonético, léxico, morfosintáctico, pragmático u otros. En este estudio nos detendremos en los elementos de carácter gramatical que aparecen a lo largo de la encuesta, destacando dos aspectos: en primer lugar, el papel del componente morfosintáctico frente a otros, como el fonético o el léxico, que, como ya hemos advertido, han acaparado el foco de los hablantes en la percepción y descripción lingüísticas. En segundo lugar y de manera complementaria, ofrece interés observar la propia consideración por parte de hablantes de diverso origen social de los diferentes fenómenos gramaticales, dialectales y no dialectales, cuya complejidad y nivel de abstracción dificultan su análisis y, por lo tanto, su definición y denominación.

La muestra, que está constituida por un total de 114 informantes, ha sido diseñada con principios de representatividad. Así, se ha buscado una distribución geográfica proporcional, de forma que todos los municipios (30) y todas las localidades (41) investigadas están representadas, pero ponderando el número de informantes de cada una de acuerdo con sus datos demográficos. Asimismo, y aunque no llevaremos a cabo un estudio de tipo variacional que tenga en cuenta las variables independientes, el conjunto de entrevistados exhibe un perfil sociolingüístico equilibrado en lo que concierne al sexo, edad y nivel sociocultural: cuenta, por lo tanto, con representación homogénea de todas las variables.

En lo que concierne a la caracterización lingüística del área estudiada, el oriente de Cantabria, al contrario que el occidente, no posee estudios y referencias suficientes para establecer una posición clara en el conjunto de modalidades del español norteño. Así, mientras que el oeste de la región se considera una extensión del leonés con debilitamiento de los rasgos de este dialecto, el este es diagnosticado como la "confluencia de matices dialectales sin características peculiares" (García-Lomas 1949: 31-32). Se trata de una afirmación que hay que atribuir más al desinterés por la especificación dialectal de la zona y a un desconocimiento de ella desde antiguo que a una verdadera situación de

10 Previamente se habían formulado las preguntas “¿En Cantabria se habla bien o mal?” y “¿Cree usted que hay personas que hablan demasiado "fino"?". 
indefinición lingüística, de tierra de nadie dialectal. Lo cierto es que, dejando de lado la presencia de algunas zonas de marcada personalidad lingüística, zonas relativamente aisladas como los valles pasiegos, y el evidente sustrato leonés que se percibe a través de una serie de fenómenos, los estudios más antiguos y más recientes coinciden en incluir esta zona dentro del complejo de dialectos castellanos. Así, en 1906, Menéndez Pidal (1962 [1906]: 12) ya afirmaba que "tan castellanizada está desde antiguo esta parte oriental del antiguo reino, que no será fácil hallar modernamente algún rasgo fonético que convenga poco más o menos con el límite antiguo"11.

En observaciones más actuales se percibe esta misma situación: se trata de un área con rasgos propios pero perteneciente, sin duda, al llamado español centro-peninsular norteño o español castellano (Moreno Fernández, 2000, 2010), que continúa siendo dominante por su tradición, prestigio y prescripción (Amorós et al., 2013) aunque "en un tiempo ciertamente muy corto en el mundo hispano parece haberse girado de una concepción del castellano peninsular como dialecto primario del español [...] a una concepción más suelta y comprehensiva" (Demonte, 2003: 10). Así, como confirma Amorós Negre (2013), se ha producido un desarrollo notable de los estándares regionales específicos, que, a pesar de su falta de codificación explícita, funcionan como paradigmas de referencia y corrección y, en definitiva, como prototipos lingüísticos que conviven con el tradicionalmente prescrito.

Las comunidades de habla de uno de los centros de una lengua pluricéntrica son conscientes de tener una lengua común como el español, pero reconocen, por un lado, una serie de formas lingüísticas características y diferenciadoras del grupo, tienen una apreciación positiva de esas formas y no las ven inferiores a otras variantes ni a la variante normativa (Bierbach 2000: 149, apud Morgenthaler García 2008: 184).

En el caso del oriente de Cantabria, todos los indicios señalan que los fenómenos característicos del leonés van diluyéndose a medida que nos alejamos del foco más dialectal, centro de Asturias, y nos dirigimos hacia el oriente. Por eso, es esperable que la zona que aquí estudiamos no presente tanta nitidez en sus rasgos dialectales como otras. En resumen, posiblemente hubo un momento en el que perteneció al área de influencia del leonés, pero las características actuales del habla la incluyen sin duda en el dominio del castellano (Fernández Juncal 1998). Empleando la expresión de Moreno Fernández (2000: 38), se trata de una zona periférica del español pero que se caracteriza por su conservadurismo lingüístico y está también sujeta a la norma culta castellana.

\section{RESULTADOS DE LA INVESTIGACIÓN}

\subsection{Análisis cuantitativo}

Como adelantábamos en el apartado anterior, las encuestas trataban de recabar información sobre determinados asuntos vinculados a la norma, las hablas limítrofes y la propia situación sociolingüística de la comunidad. Las respuestas a estas interrogantes

11 Menéndez Pidal (1962: 16). Esta aseveración está ratificada en otros trabajos como los de Rodríguez Castellano (1954), Zamora Vicente (1979) o Neira (1989). 
remitían en muchas ocasiones a consideraciones de tipo extralingüístico ${ }^{12}$. De hecho, se producía habitualmente una doble interpretación de la demanda: las respuestas a la pregunta “¿por qué?” aludían, por una parte, a la causa del hecho ("Las mujeres hablan mejor porque han recibido más educación”) y, por otra parte, a la manifestación del hecho ("las mujeres hablan mejor porque pronuncian mejor"). Son estas últimas opiniones, con información acerca de fenómenos que se producen en diferentes niveles de la lengua, las que constituyen nuestro foco de análisis y las que se detallarán en las consiguientes tablas.

En el cuadro 1 se puede observar el desglose de este tipo de respuestas en función de su adscripción a los diferentes niveles lingüísticos (fonético, léxico o morfosintáctico) o a un cuarto apartado, que recoge apreciaciones de carácter general o pertenecientes a otras subdisciplinas de carácter lingüístico, como la pragmática o la ortografía ${ }^{13}$.

\begin{tabular}{|c|c|c|c|c|c|c|}
\hline & & FONÉTICA & LÉXICO & GRAMÁTICA & OTROS & total \\
\hline \multirow{5}{*}{ NORMA } & $\begin{array}{l}\text { Incorrección en } \\
\text { Cantabria }\end{array}$ & 24 & 19 & 79 & 13 & 135 \\
\hline & $\begin{array}{l}\text { Rasgos de habla } \\
\text { fina }\end{array}$ & 21 & 18 & 2 & 11 & 52 \\
\hline & $\begin{array}{l}\text { Corrección } \\
\text { externa }\end{array}$ & 4 & 13 & 14 & 1 & 32 \\
\hline & $\begin{array}{l}\text { Rasgos de } \\
\text { los medios de } \\
\text { comunicación }\end{array}$ & 20 & 31 & 6 & 27 & 84 \\
\hline & $\begin{array}{l}\text { Diferencia } \\
\text { hablado-leído }\end{array}$ & 77 & 14 & 2 & 21 & 114 \\
\hline & & & & & subtotal & 417 \\
\hline \multirow{3}{*}{$\begin{array}{c}\text { HABLAS } \\
\text { LIMÍTROFES }\end{array}$} & Asturianos & 68 & 44 & 20 & 15 & 147 \\
\hline & Castellanos & 19 & 6 & 6 & 16 & 47 \\
\hline & Vascos & 50 & 29 & 24 & 27 & 130 \\
\hline & & & & & subtotal & 324 \\
\hline \multirow{6}{*}{$\begin{array}{c}\text { VARIACIÓN } \\
\text { SOCIOLING } \\
\text { INTERNA }\end{array}$} & Variable sexo & 3 & 8 & 0 & 10 & 21 \\
\hline & Variable edad & 7 & 19 & 3 & 10 & 39 \\
\hline & Profesión & 0 & 5 & 0 & 1 & 6 \\
\hline & Rural - Urbano & 4 & 10 & 2 & 5 & 21 \\
\hline & Costa- Interior & 14 & 7 & 1 & 0 & 22 \\
\hline & & & & & subtotal & 109 \\
\hline & & 311 & 223 & 159 & 157 & 850 \\
\hline
\end{tabular}

Cuadro 1. Clasificación de la ejemplificación de tipo metalingüístico

12 En palabras de Coseriu (1993: 63): "valoran otros aspectos del hablar en cuanto acción, en cuanto a manifestación del ser, del hablante, de la cultura del hablante, etc.”.

13 Por tratarse, por lo tanto, de datos misceláneos y a efectos de realizar el análisis cuantitativo, esta columna no entrará en el análisis estadístico futuro con los otros tres niveles. 
A partir de las 13 interrogantes se han recogido 850 ejemplos, repartidos de manera irregular en los diferentes bloques. Son los que requieren información sobre la norma o sobre las hablas limítrofes los más productivos (49,1\% y 38,1\%) y menos (solo un 12,8\%), el que indaga sobre la variación interna de la comunidad, donde precisamente abundan más las manifestaciones extralingüísticas, que evalúan más los diferentes grupos analizados (hombres y mujeres, jóvenes y adultos, habitantes de medio rural frente a habitantes de medio urbano, etc.) que el habla que los caracteriza.

En lo que respecta a la presencia de los diferentes niveles ${ }^{14}$, es el fónico el que acapara mayor número de respuestas $(36,6 \%)$, seguido por el léxico $(26,2 \%)$ y finalmente por el morfosintáctico (18,7\%). Podríamos pensar que algunos de los ítems, por su propio contenido, conducen a una mayor aparición de rasgos fonéticos: es el caso de la pregunta que cuestiona cómo detectar si alguien está leyendo o está hablando sin apoyo de texto y otra, muy ligada a esta primera, relativa a la diferencia existente entre el habla de los profesionales de los medios de comunicación y el habla de la calle. Incluso eliminando ese tipo de consultas, los fenómenos de ese nivel lingüístico destacan notablemente sobre los demás y son la respuesta mayoritaria en 6 entradas en total.

La misma distribución de datos es la que hallamos en la encuesta anterior de 1996 (Fernández Juncal 2000): sigue siendo el nivel fónico el que recibe más ejemplificación, seguido por el léxico y muy minoritariamente por el componente morfosintáctico, por lo que podríamos inferir una cierta tendencia.

Los datos de la encuesta relativos al componente morfosintáctico confirman su posición en los estudios de creencias, con una presencia notablemente inferior a los otros dos niveles de lengua. Así, su aparición es nula o casi nula en algunos de los ítems formulados: rasgos que definen el habla de las mujeres y de las profesiones donde mejor y peor se habla, distinción entre la modalidad de la costa y la del interior, el medio rural y el medio urbano. A esto se añade que en la mayoría del resto de las preguntas es el nivel con menor ejemplificación, siempre por detrás de lo fónico y del léxico ${ }^{15}$.

No obstante, hay dos ítems cuyos resultados contrarían la idea de que la conciencia morfosintáctica está lastrada por la dificultad intrínseca de su propio contenido. Se trata de las preguntas “¿Puede poner algún ejemplo de algo que se diga mal en Cantabria?” y “LLe han corregido a usted alguna vez? ¿En qué circunstancia?”. Para estos interrogantes, y en uno de ellos de manera destacada, la mayoría de los casos citados cae en la órbita de la morfosintaxis. Si analizamos la formulación de estas dos consultas frente al resto, la diferencia estriba en que aparece el concepto de corrección (e incorrección). Mientras, en los demás ítems, las preguntas están enunciadas en términos de diferencia, lo cual no implica necesariamente una evaluación de grado o escala y menos una posición entre posiciones antagónicas. Parece, por consiguiente, que, puestos en posición de tener que elegir entre categorías contrarias, como es el caso, los hablantes sí citan fenómenos propios del nivel morfosintáctico, donde se produce esa oposición.

Convendría someter estos resultados globales a un análisis estadístico de forma que podamos comprobar si la distribución de contestaciones es azarosa o si se puede demos-

14 En negrita, las respuestas mayoritarias.

15 Es también la misma situación que encuentran Anglejan y Tucker (1973) en Montreal. 
trar una correlación entre el tipo de pregunta y el reparto de respuestas en los tres grupos mencionados, Aplicando la prueba de ji-cuadrado $\left(\chi^{2}\right)$ a los porcentajes del recuento, detallamos en el cuadro 2 los resultados de significación para cada una de las 13 entradas:

\begin{tabular}{|c|c|c|c|c|c|c|c|}
\hline ENTRADA & & & FONÉTICA & LÉXICO & GRAMÁTICA & total & VALOR p \\
\hline \multirow{10}{*}{ NORMA } & \multirow{2}{*}{$\begin{array}{l}\text { Incorrección } \\
\text { en Cantabria }\end{array}$} & Recuento & 24 & 19 & 79 & 122 & \multirow{2}{*}{$0,000 * *$} \\
\hline & & Frec esperada & 47,2 & 54.8 & 20,0 & 122,0 & \\
\hline & \multirow{2}{*}{$\begin{array}{l}\text { Rasgos de } \\
\text { habla fina }\end{array}$} & Recuento & 21 & 18 & 2 & 41 & \multirow{2}{*}{$0,002 *$} \\
\hline & & Frec esperada & 15.9 & 18,4 & 6,7 & 41,0 & \\
\hline & \multirow{2}{*}{$\begin{array}{l}\text { Corrección } \\
\text { externa }\end{array}$} & Recuento & 4 & 13 & 14 & 31 & \multirow{2}{*}{$0,000 * *$} \\
\hline & & Frec esperada & 12,0 & 13,9 & 5,1 & 31,0 & \\
\hline & \multirow{2}{*}{$\begin{array}{l}\text { Rasgos de } \\
\text { los medios de } \\
\text { comunicación }\end{array}$} & Recuento & 20 & 31 & 6 & 57 & \multirow[t]{2}{*}{0,111} \\
\hline & & Frec esperada & 22,1 & 25,6 & 9,3 & 57,0 & \\
\hline & \multirow{2}{*}{$\begin{array}{l}\text { Diferencia } \\
\text { hablado-leído }\end{array}$} & Recuento & 77 & 14 & 2 & 93 & \multirow{2}{*}{$0,000 * *$} \\
\hline & & Frec esperada & 36,0 & 41,8 & 15,2 & 93,0 & \\
\hline \multirow{6}{*}{$\begin{array}{c}\text { HABLAS } \\
\text { LIMÍTROFES }\end{array}$} & \multirow{2}{*}{ Asturianos } & Recuento & 68 & 44 & 20 & 132 & \multirow{2}{*}{$0,025 *$} \\
\hline & & Frec esperada & 51,1 & 59,3 & 21,6 & 132,0 & \\
\hline & \multirow{2}{*}{ Castellanos } & Recuento & 19 & 6 & 6 & 31 & \multirow{2}{*}{$0,000 * *$} \\
\hline & & Frec esperada & 12,0 & 13,9 & 5,1 & 31 & \\
\hline & \multirow{2}{*}{ Vascos } & Recuento & 50 & 29 & 24 & 103 & \multirow{2}{*}{$0,003 *$} \\
\hline & & Frec esperada & 39,9 & 46,3 & 16,8 & 103,0 & \\
\hline \multirow{10}{*}{$\begin{array}{l}\text { VARIACIÓN } \\
\text { SOCIOLING. } \\
\text { INTERNA }\end{array}$} & \multirow{2}{*}{ Var. sexo } & Recuento & 3 & 8 & 0 & 11 & \multirow{2}{*}{$0,000 * *$} \\
\hline & & Frec esperada & 4,3 & 4,9 & 1,8 & 11,0 & \\
\hline & \multirow{2}{*}{ Var. edad } & Recuento & 7 & 19 & 3 & 29 & \multirow{2}{*}{$0,000 * *$} \\
\hline & & Frec esperada & 11,2 & 13,0 & 4,7 & 29,0 & \\
\hline & \multirow{2}{*}{ Profesión } & Recuento & 0 & 5 & 0 & 5 & \multirow{2}{*}{$0,000 * *$} \\
\hline & & Frec esperada & 1,9 & 2,2 & 0,8 & 5,0 & \\
\hline & \multirow{2}{*}{$\begin{array}{l}\text { Rural - } \\
\text { Urbano }\end{array}$} & Recuento & 4 & 10 & 2 & 16 & \multirow{2}{*}{$0,002 *$} \\
\hline & & Frec esperada & 6,2 & 7,2 & 2,6 & 16,0 & \\
\hline & \multirow{2}{*}{ Costa- Interior } & Recuento & 14 & 7 & 1 & 22 & \multirow{2}{*}{$0,000 * *$} \\
\hline & & Frec esperada & 8,5 & 9,9 & 3,6 & 22,0 & \\
\hline & \multirow{2}{*}{ TOTAL } & Recuento & 311 & 223 & 159 & 693 & \\
\hline & & Frec esperada & 311,0 & 223,0 & 159,0 & & \\
\hline
\end{tabular}

*significativo al nivel $\mathrm{p}<0,05 ; * *$ significativo al nivel $\mathrm{p}<0,001$

Cuadro 2. Tabla de contingencia. Tipo de entrada-nivel lingüístico 
Si observamos la última columna de la tabla de contingencia, donde se precisan los valores de significación de $\chi^{2}$, se aprecia que en todos los casos excepto uno (rasgos que caracterizan el habla de los medios de comunicación) se descarta la hipótesis nula y se ratifica la correlación entre el tipo de cuestión planteada y las expectativas de que la ejemplificación se decante por uno de los tres niveles de la lengua analizados; es decir, se establece la dependencia o relación de ambas variables. Destaca además que el grado de probabilidad refleja, en la mayoría de los casos, un valor $\mathrm{p}<0,001$, que resulta muy significativo, también en los dos ítems que acumulan más respuestas de tipo morfosintáctico.

Considerando los antecedentes y los datos estadísticos, podríamos explicar esta correlación en el siguiente sentido: existe para la gramática (en el sentido restrictivo del término) una concepción más exigente, menos tolerante en términos de aceptabilidad mientras que en otros niveles, el fonético o el léxico, es más arduo establecer un canon de corrección.

La variación léxica se caracteriza precisamente por la dificultad en establecer patrones de referencia (Borrego Nieto 1994), también en la variedad estándar (Amorós et al. 2012). Así, el acervo léxico de una variedad se multiplica, diverge y se solapa, y consecuentemente la evaluación del uso gira en torno a valores como la precisión, la adecuación o la coherencia estilística, sin necesidad de anular por prescripción elementos del repertorio.

Algo semejante ocurre con la variación fonética: parece superada ya la idea de que se trata de un nivel con ventajas indudables para el análisis sociolingüístico. De esa forma, se ha pasado de una consideración de las variables fónicas como fácilmente cuantificables a una concepción en la que no es tan sencillo tratar como unidades discretas lo que son en realidad unidades de continuum, y, por lo tanto, también complejas de jerarquizar. Así se entiende que en la definición del estándar español no se incluya la prescripción fonética. De hecho, Demonte (2003: 3) alude a esta ausencia como atributo de los estándares en general

Poder ser entendido por el mayor número posible de los hablantes (Crystal) ha de ser un criterio de 'estandaridad' para el supradialecto escogido. Este requisito tiene consecuencias en cuanto a los aspectos de las lenguas relevantes para la delineación del estándar. Implica, por caso, que los aspectos prosódicos -la entonación, el ritmo acentual-- no serán centrales en tal delineación, salvo que la variedad estándar se identifique claramente con un dialecto geográfico.

y del estándar español en particular, "donde los acentos no se manifiestan de forma llamativa, aunque persisten rasgos, particularmente fonéticos y prosódicos, que identifican la zona geográfica a la que pertenece el hablante" (Demonte: 2003: 7).

Por su parte, la variación sintáctica, tan poco estudiada, tan compleja metodológicamente, presenta características que pueden esclarecer los resultados de la encuesta. En primer lugar, la variación se manifiesta en muchas ocasiones de manera dicotómica. En Fernández Juncal y Amorós Negre (2014) hablamos de polarización y tensión normativa, para dar cuenta de una situación similar a la descrita: sometidos a una serie de estímulos lingüísticos que recogían diversos fenómenos gramaticales del español actual ${ }^{16}$, los informantes ${ }^{17}$ los evaluaban en su mayoría con los extremos de una escala y no con puntuaciones interme-

16 Orden de clíticos, usos pronominales (leísmo, laísmo y loísmo), falta de concordancia sujeto-verbo, marcas referenciales en adverbios, queísmo, dequeísmo y quesuismo, flexión verbal, etc.

17 Se trataba de informantes menores de 30 años con estudios superiores. 
dias. Así, es perceptible en general una cierta correspondencia entre la evaluación de los hablantes y la de las instituciones normativas, tal y como se observa en la coincidencia de valoraciones de los fenómenos más polarizados, bien por su prestigio manifiesto, bien por su estigmatización. Solo aquellos fenómenos en los que la prescripción no es clara se sitúan en los valores intermedios; es decir, los casos más numerosos en estos intervalos centrales responden a fenómenos en los que se produce una contradicción evidente entre la norma prescrita por las gramáticas y la norma empírica del hablante, que constata que tales usos están presentes en el habla de las personas cultas, incluso en situaciones formales ${ }^{18}$. En la investigación presente la presencia de fenómenos estigmatizados (tal como se requiere por la formulación de la pregunta) subraya el valor dicotómico que muestra la variación gramatical y que la distingue de la variación fonética y léxica, cuya norma es más difusa e imprecisa.

\subsection{Análisis cualitativo}

En la investigación que nos ocupa encontramos también esa diferenciación entre fenómenos polarizados y fenómenos con valoraciones intermedias. A lo largo de las entrevistas se aportó información de tipo evaluativo sobre distintos fenómenos que se producen en este nivel: por una parte, aquellos que se consideran estigmatizados al separarse de la norma ${ }^{19}$ $\mathrm{y}$, por otra parte, aquellos que sirven para diferenciar dialectos o sociolectos. Pertenecen al primer grupo los resumidos en el cuadro $3^{20}$ :

\begin{tabular}{|l|l|c|}
\hline $\begin{array}{l}\text { FENÓMENO } \\
\text { MORFOSINTÁCTICO }\end{array}$ & CITAS & $\begin{array}{c}\text { Número de } \\
\text { citaciones }\end{array}$ \\
\hline Leísmo & $\begin{array}{l}\text { Leísmo total/ a veces confundimos le y lo / se usa } \\
\text { demasiado el leísmo / la y le se dicen de pena/ el le } \\
\text { y el la }\end{array}$ & 25 \\
\hline $\begin{array}{l}\text { Uso de condicional por } \\
\text { subjuntivo }\end{array}$ & $\begin{array}{l}\text { Si podría... / utilizar el condicional cuando no } \\
\text { corresponde / si tendría.../ si vendría.../ verbos en } \\
\text { condicional / pondrían / el condicional en situaciones } \\
\text { en que no es necesario / habría en lugar de hubiera o } \\
\text { hubiese / se usa el condicional en lugar del imperfecto/ } \\
\text { si te contaría.../ le dijo que vendría / estaría / si habría } \\
\text { / usar el condicional indebidamente }\end{array}$ & 23 \\
\hline Laísmo & $\begin{array}{l}\text { Sustituir el la como complemento directo/ somos } \\
\text { laístas / laísmo }\end{array}$ & 17 \\
\hline Sufijo -uco & $\begin{array}{l}\text { Hijuco /adjetivos terminados en -uco/ Se usa mucho } \\
\text { el diminutivo - -uco y - uca / chicuco / modismos como } \\
\text { niñuco no están en al Academia pero forman parte de } \\
\text { la cultura / a los de fuera les hace gracia lo de casuca }\end{array}$ & 6 \\
\hline
\end{tabular}

18 Es lo que ocurre con el quesuismo y el queísmo, las formas analógicas de algunos verbos irregulares como andar, la combinación de adverbios de lugar con pronombres tónicos [Detrás mío y delante de mi] y las concordancias ad sensum entre sujetos con sustantivo colectivo y verbos en plural.

19 Respuestas a las preguntas sobre incorrección en Cantabria y la corrección externa.

20 Por el interés que presentan en la enunciación y descripción de los diferentes fenómenos, incluimos una selección de citas textuales recogidas en la encuesta. 


\begin{tabular}{|l|l|c|}
\hline $\begin{array}{l}\text { FENÓMENO } \\
\text { MORFOSINTÁCTICO }\end{array}$ & CITAS & $\begin{array}{c}\text { Número de } \\
\text { citaciones }\end{array}$ \\
\hline haiga & Dicen haiga / haiga por haya/ & 3 \\
\hline Orden de clíticos & Me se ocurre / Gente mayor dice se me / me se por se me / & 3 \\
\hline Dequeísmo & Decimos de que / dequeísmo / & 2 \\
\hline Contra más & Contra más... & 2 \\
\hline Otros & $\begin{array}{l}\text { Loísmo } \\
\text { La con nombres de mujeres } \\
\text { vos por } \text { os } \\
\text { Queísmo } \\
\text { hais por habéis } \\
\text { a veces se utilizan los tiempos compuestos cuando son } \\
\text { simples }\end{array}$ & \\
\hline
\end{tabular}

Cuadro 3. Detalle de fenómenos lingüísticos citados (respuestas a preguntas sobre corrección)

Por otra parte, pormenorizamos en el cuadro 4 los rasgos gramaticales que sirven para la descripción de otros dialectos y sociolectos:

\begin{tabular}{|c|c|c|c|}
\hline $\begin{array}{l}\text { DIALECTOS- } \\
\text { SOCIOLECTOS- } \\
\text { ESTILOS }\end{array}$ & $\begin{array}{l}\text { FENÓMENO } \\
\text { MORFOSINTÁCTICO }\end{array}$ & CITAS & $\begin{array}{l}\text { Número } \\
\text { de } \\
\text { citaciones }\end{array}$ \\
\hline \multirow{5}{*}{ ASTURIANOS } & $\begin{array}{l}\text { Posposición } \\
\text { pronombres }\end{array}$ & $\begin{array}{l}\text { Paselo yo (posponen el artículo) / Una } \\
\text { forma diferente de construir la frase / } \\
\text { préstame / expresiones que le dan la } \\
\text { vuelta al orden / pronombre después } \\
\text { del verbo / el pronombre va al final / } \\
\text { cambian el reflexivo / díjome / mala } \\
\text { utilización de los artículos }\end{array}$ & 10 \\
\hline & ye & Usan ye / ye por es / ¿Qué ye? & 9 \\
\hline & Tiempos verbales & $\begin{array}{l}\text { hablan en pasado / en lugar de “¿quién } \\
\text { lo ha dicho?" dicen “¿quién lo dijo?" / } \\
\text { ¿te mancaste? / acaban el pasado / usan } \\
\text { pasados simples / el pretérito }\end{array}$ & 8 \\
\hline & -ín/a & vaquina / guapina / la terminación-nín & 3 \\
\hline & Otros: & $\begin{array}{l}\text { ¿lo qué? } \\
\text { ¿lo cuálo? } \\
\text { construyen mal alguna frase }\end{array}$ & 2 \\
\hline \multirow{3}{*}{ CASTELLANOS } & Leísmo & También tienen leísmo & 2 \\
\hline & Uso trans. de caer & Lo caes/ lo he caído & 2 \\
\hline & Otros & $\begin{array}{l}\text { Chiquito } \\
\text { Ponen artículo a las personas como en } \\
\text { la Pepa }\end{array}$ & 2 \\
\hline
\end{tabular}




\begin{tabular}{|c|c|c|c|}
\hline $\begin{array}{l}\text { DIALECTOS- } \\
\text { SOCIOLECTOS- } \\
\text { ESTILOS }\end{array}$ & $\begin{array}{l}\text { FENÓMENO } \\
\text { MORFOSINTÁCTICO }\end{array}$ & CITAS & $\begin{array}{l}\text { Número } \\
\text { de } \\
\text { citaciones }\end{array}$ \\
\hline \multirow{4}{*}{ VASCOS } & Pues pospuesto & $\begin{array}{l}\text { Oye, pues / el pues al final / Ostia, pues } \\
\text { / Anda, pues / A la mañana, pues/ }\end{array}$ & 13 \\
\hline & Condicional & $\begin{array}{l}\text { mal uso de condicional también / en } \\
\text { ocasiones, el condicional / }\end{array}$ & 3 \\
\hline & $\begin{array}{l}\text { Uso de artículo con } \\
\text { nombre propio }\end{array}$ & $\begin{array}{l}\text { El Iñaki, la Arantza } \\
\text { La y el con sustantivo propio }\end{array}$ & 2 \\
\hline & Otros & $\begin{array}{l}\text { Al de... } \\
\text { en lugar de "fuimos fulano y yo", ellos } \\
\text { dicen "fuimos fulano y los dos". } \\
\text { Hablan con infinitivos } \\
\text { Ninguno diríamos -ita }\end{array}$ & 4 \\
\hline \multirow[t]{2}{*}{ JÓVENES } & Corrección gramatical & $\begin{array}{l}\text { Cometen menos errores gramaticales / } \\
\text { construyen mejor }\end{array}$ & 4 \\
\hline & Otros & $\begin{array}{l}\text { Repiten fórmulas del periodismo } \\
\text { deportivo: hablan en } 3^{\mathrm{a}} \text { persona y con } \\
\text { plural mayestático } \\
\text { Usan super- y mega- }\end{array}$ & 2 \\
\hline MEDIO RURAL & Me se & $\begin{array}{l}\text { Y hay gente mayor que dice "me se } \\
\text { cayó" }\end{array}$ & 1 \\
\hline \multirow{2}{*}{$\begin{array}{l}\text { MEDIOS DE } \\
\text { COMUNICACIÓN }\end{array}$} & Corrección gramatical & $\begin{array}{l}\text { Por la construcción de frases / el } \\
\text { locutor es más correcto en la estructura } \\
\text { /la gramática se suele mantener }\end{array}$ & 3 \\
\hline & Otros & $\begin{array}{l}\text { en la tele hay problema de "detrás } \\
\text { tuya", frases mal formadas. Mejor en } \\
\text { la radio }\end{array}$ & 1 \\
\hline LECTURA & $\begin{array}{l}\text { Diferencias } \\
\text { estructurales }\end{array}$ & $\begin{array}{l}\text { en las construcciones gramaticales y la } \\
\text { forma de expresarse / } \\
\text { la forma de construir frases }\end{array}$ & 2 \\
\hline
\end{tabular}

Cuadro 4. Detalle de fenómenos lingüísticos citados (respuestas a preguntas sobre dialectos, sociolectos, estilos)

Advertimos en primer lugar que hay una serie de fenómenos que se repiten en las dos tablas. Es el caso del leísmo o el empleo de condicional por subjuntivo aunque con mucha menos presencia que en el cuadro 3. En este último abundan más los rasgos gramaticales con marca diatópica, no necesariamente enfrentados con la norma, como el uso de pues pospuesto, recogido, por ejemplo, en la Nueva gramática de la lengua española $(\$ 46.12 \mathrm{~m})$ : "Como expresión ilativa, pues admite dos usos. En el primero es tónica y se puede considerar adverbio [...]. Se caracteriza por aparecer al final de una oración”. 
También, con marca de arcaizante, es incluida en la misma obra (§16.7e) la posposición de los clíticos con formas personales, que nuestros informantes relacionan, con buen tino, con la variedad hablada en Asturias:

No obstante, la enclisis a las formas personales del verbo (díjolo, violas, contestole) se documenta hasta el siglo XX tanto en el español europeo como en el americano, con más frecuencia a principio de oración o tras pausa [...]. Se detecta también su empleo en los registros más formales de la expresión oral.

Lo mismo ocurre con la preferencia por el pretérito perfecto simple frente al compuesto, cuyo empleo en esa misma área es también mencionado (\$23.7c): "En estas zonas [el noroeste de España y en las islas Canarias], el pretérito perfecto simple sustituye al compuesto con independencia del valor temporal o aspectual de la acción".

Confirmamos en gran medida la hipótesis de que tratamos, en el caso de la conciencia gramatical, con dos tipos de evaluación: por una parte, existen una serie de rasgos gramaticales que se desvían de la norma, que están estigmatizados, muy presentes en la percepción de los hablantes y con un marchamo negativo, muy polarizado respecto a la variante normativa. Son estos los que aparecen de manera mayoritaria cuando la pregunta gira en torno al concepto de corrección. Por otra parte, existe otro tipo de fenómenos, que la norma generalmente asimila (a veces con marca, pero asimila), ante los cuales el hablante emite un juicio menos taxativo, más condescendiente. Son los que surgen cuando la consulta se hace en términos de diferencia, pero, eso sí, ocupando una posición secundaria respecto a otros fenómenos más perceptibles en otros niveles de la lengua: el fonético y el léxico.

\section{CONCLUSIONES}

Si consideramos la conciencia de los hablantes respecto a diferentes rasgos lingüísticos, es el nivel fónico el que concentra el mayor porcentaje de respuestas, seguido por el nivel léxico. Este resultado concuerda con algunos de los trabajos de la literatura citada, aunque en ocasiones se ha podido observar una alternancia en la posición ocupada por ambos. En este caso, la preponderancia de los rasgos fónicos coincide con estudios previos, pero puede venir también determinada por la configuración del cuestionario, que incide en aspectos que podrán focalizar la atención en cuestiones como la pronunciación o la entonación. De cualquier forma, se trata de dos niveles de lengua que poseen rasgos comunes. En primer lugar, resulta indudable que se encuentran en un grado de conciencia de los hablantes superior a fenómenos que se producen en otros niveles. En segundo lugar, si los definimos en su relación con la norma, ambos son más accesibles, más obvios en la variación interna de los elementos que los configuran y, por esa misma razón, presentan mayor indefinición de una norma propia.

Por su parte, el nivel morfosintáctico, de gran complejidad de análisis, presenta, como sospechábamos, una posición exigua respecto al fonético o al léxico. Sin embargo, hay cuestiones que invierten esa posición y hacen que la conciencia sobre la variación gramatical sea más notoria: son los ejemplos de desviaciones de la norma gramatical los más numerosos cuando el juicio del hablante se sitúa en términos de polarización normativa. Ahí, las variantes gramaticales opuestas a la norma, mucho más definida que la de otros niveles, se 
manifiestan con más claridad. De hecho, la equipolencia entre variedades y variantes tanto estándares como no estándares, defendida por la mayor parte de lingüistas, no se refleja en las actitudes manifestadas por el grueso de la población, que, cuando se les cuestiona a este respecto, establece una jerarquía de usos lingüísticos en la cual las variantes codificadas resultan privilegiadas y prestigiadas frente a las que se escapan de la norma, que son consideradas de manera negativa.

En el análisis cualitativo se produce la misma segregación de resultados: cuando la interrogación gira en torno a los rasgos que permiten distinguir diferentes colectivos, encontramos fenómenos que la propia norma incluye dentro del paradigma. Por el contrario, cuando la pregunta se formula en términos de incorrección, lo que implica la superioridad de uno de los elementos en conflicto y la inferioridad del otro u otros, además de, como hemos visto, incrementar las cifras de citación, se mencionan variantes claramente estigmatizadas, desechadas por el canon de corrección.

Nos movemos, por consiguiente, entre dos nociones que entroncan con la tradición platónica: jerarquía y diferencia, cuya relación se expone y desarrolla ya en el Parménides (Zistakis 2006). Se trata de conceptos con incidencia directa en otros que definen la posición y conciencia de comunidades de habla que se hallan en la periferia de los modelos de lengua: diversidad y unidad, cambio y mantenimiento lingüísticos.

Estos resultados abren un camino hacia la investigación sobre la incidencia de la conciencia lingüística en el devenir de estos fenómenos polarizados, más inestables y más sometidos, por lo tanto, a la presión del colectivo y de sus líderes en la configuración de la norma interna de la comunidad.

\section{Referencias bibliográficas}

Agheyisi, R. y J. Fishman (1970). "Language attitude studies: A brief survey of methodological approaches". Anthropological Linguistics 12 (5), pp. 137-157.

Ajzen, I. \& Fishbein, M.. (1980). Understanding attitudes and predicting social behavior. New Jersey: Prentice-Hall, Inc

Alvar, M. (1977), “Actitud del hablante y sociolingüística”. En Lapesa, R. (coord.). Comunicación y lenguaje. Madrid: Karpos, pp. 87-105.

Amorós Negre, C. (2013). Descripción y prescripción en un estándar pluricéntrico. Análisis del paradigma de los relativos en las normas lingüísticas del español (tesis doctoral). Universidad de Salamanca.

Amorós Negre, C., C. Fernández Juncal, N. Hernández Muñoz y E. Prieto de los Mozos (2012)."Difficulties in defining the standard Spanish lexicon". En Muhr, R. (ed.). Non-dominating Varieties of pluricentric Languages. Getting the Picture. In memory of Prof. Michael Clyne. Viena: Peter Lang Verlag, pp. 61-80.

Anglejan, A. y G. Tucker (1973). "Sociolinguistic correlates of speech style in Québec". En Shuy, R. y R. Fasold (eds.). Language attitudes: Current, trends and prospects. Washington: Georgetown University Press, pp. 1-27.

Bierbach, M. (2000). "Spanisch - eine plurizentrische Sprache? Zum Problem von norma culta und Varietät in der hispanophonen Welt". Vox romanica 59, pp. 143-170.

Borrego Nieto, J. (1981). Sociolingüística Rural. Salamanca: Universidad de Salamanca.

Borrego Nieto, J. (1992). "Actitudes y prejuicios lingüísticos: la norma interna del hablante”, en J. A. Bartol et al. (eds.). Estudios Filológicos en Homenaje a Eugenio de Bustos Tovar. Salamanca: Universidad de Salamanca, pp. 121135. 
Borrego Nieto, J. (1994). "Dificultades para el estudio sociolingüístico del léxico". En Garza Cuarón, B. et al. (coords.). II Encuentro de lingüistas y filólogos de España y México. Salamanca: Universidad de Salamanca/Junta de Castilla y León, pp. 119-132.

Boyer, H. (1991). Éléments de sociolinguistique. Paris: Dunod.

Buzón García, J.M., M.B. Gómez Devís y J.R. Gómez Molina (2017). Actitudes lingüísticas en Valencia y su área metropolitana. Estudio longitudinal y análisis de tendencias. Valencia: Tirant Humanidades.

Caravedo, R. (2014). Percepción y variación lingüística. Enfoque sociocognitivo. - Madrid/Frankfurt: Iberoamericana/Vervuert.

Cazacu, R. (1968). "Sur la notion de 'interdialecte'". En Quilis, A. et al. (eds.). XI Congreso Internacional de Lingüística y Filología Románicas (Madrid, 1965). Madrid: Consejo Superior de Investigaciones Científicas, pp. 1457-1465.

Cestero Mancera, A. M. y F. Paredes García (2013). "Metodología PRECAVESXXI. Proyecto para el estudio de creencias y actitudes hacia las variedades del español en el siglo XXI". <http://www. variedadesdelespanol.es/Content/Metodolog\%C3\%ADa\%20proyect o\%20PRECAVES-XXI.pdf $>$ $(20 / 12 / 16)$.

Cestero Mancera, A.M. y F. Paredes García (2015a). "Creencias y actitudes hacia las variedades normativas del español actual: primeros resultados del Proyecto PRECAVES-XXI". Spanish in Context 12/2: 255-279.

Cestero Mancera, A.M. y F. Paredes García (2015b). "Creencias y actitudes hacia las variedades del español en el siglo XXI: avance de un proyecto de investigación”. En da Hora, D. et alii (eds.). ALFAL 50 anos: contribuições para os estudos linguísticos e filológicos. João Pessoa: Ideia, pp. 652-683.

Chiquito, A. B. y M. Á. Quesada Pacheco (eds.) (2014). Actitudes lingüisticas de los hispanohablantes hacia el idioma español y sus variantes. Bergen: Universitetet i Bergen.

Cooper, R. L. y J.A. Fishman (1974). "The study of language attitudes", International Journal of the Sociology of Language, 3, pp. 5-19.

Coseriu, E. (1993). Competencia lingüistica y criterios de corrección. Apuntes del curso intensivo de perfeccionamiento dictado por el prof. Dr. Eugenio Coseriu (19 a 22 de octubre de 1987). A. Matus y J. L. Samaniego (eds.). Santiago: Pontificia Universidad Católica de Chile.

Cots, J., L. Armengol, E. Arnó, M. Irún, M. y E. Llurda (2007). La conciencia lingüística en la enseñanza de lenguas. Barcelona: Graó.

Demonte, V. (2003). "Lengua estándar, norma y normas en la difusión actual de la lengua española". Circunstancia, 1. http://www.ortegaygasset.edu/publicaciones/circunstancia/ano-i---numero-1--abril-2003/estados-de-la-cuestion/lengua-estandar--norma-y-normas-en-la-difusion-actual-de-lalengua-espanola (12-09-16).

Dik, S. (1997 [1989]). The Theory of Functional Grammar. 1.- The Structure of the Clause. Berlín/N. York: Mouton de Gruyter.

Edwards, J. (2011). Challenges in the Social Life of Language. Londres: Palgrave Macmillan.

Ehri, L. et al. (2001). "Phonemic awareness instruction helps children learn to read: Evidence from the National Reading Panel's meta-analysis". Reading Research Quarterly, 36 , pp. 250-287.

Ellis, S. (1969). "The assessment of linguistic boundaries by local dialect speakers". En Graur, A. (ed.). Actes du Xe. Congrès International de Linguistes II (Bucarest, 1967). Bucarest: Éditions de la R.S.R., pp. 110-112.

Espinosa Taset, I. (2009). "Las creencias de aprendizaje de principiantes brasileños sobre la escritura en la adquisición del E/LE”, Revista Nebrija de lingüística aplicada a la enseñanza de las lenguas 6. $<\mathrm{http}$ ://www.nebrija.com/revista-linguistica/lascreencias-de-aprendizaje-de-principiantesbrasile\%C3\%B1os-sobre-la-escrituraen-la-adquisicion-del-e-le> (19/03/17).

Fernández Juncal, C. (1998). Variación y prestigio: estudio sociolingüístico en el oriente de Cantabria. Madrid: CSIC. 
Fernández Juncal, C. (2000). Neutro de materia y metafonía en el oriente de Cantabria. Salamanca: Universidad de Salamanca.

Fernández Juncal, C. (2005). “¿Video meliora, proboque; deteriora sequor? Hechos y actitudes lingüísticas en el CHCS”. En Santos Río, L. et al. (eds.). Palabras, norma, discurso. En memoria de Fernando Lázaro Carreter. Salamanca: Universidad de Salamanca, pp. 425-432.

Fernández Juncal (en prensa). "Percepción de la norma en la modalidad centro-peninsular del español: los nuevos modelos lingüísticos". Coloquio Internacional El pluricentrismo de la cultura lingüistica hispánica y sus reflejos en los medios de comunicación masiva. Perspectivas empíricas. Rheinische Friedrich-Wilhelms-Universität Bonn (Alemania), 24-27 de octubre de 2016.

Fernández Juncal, C. y C. Amorós Negre (2014). "Polarización y tensión normativas. Actitudes hacia la norma prescrita en el español peninsular centro-septentrional". En Zimmermann, K. (ed.) Prácticas y politicas lingüísticas. Nuevas variedades, normas, actitudes y perspectivas. Frankfurt: Iberoamericana Vervuert, pp. 231-256.

García-Lomas y García-Lomas, A. (1949). El lenguaje popular de las montañas de Santander. Santander: Diputación Provincial de Santander.

Giles, H. y E. Bouchard Ryan (1982). Attitudes towards Language Variation. London, Arnold.

Givón, T. (2001, nueva ed.). Syntax. I. Amsterdam/ Philadelphia: J. Benjamins.

Haenni, R. (1999). The Case of Estuary English: Supposed Evidence. (tesis doctoral, Univ. of Basel). http://www.phon.ucl.ac.uk/home/estuary/haenni1999.pdf (13-10-16).

Hansen, K. (2007). H-Dropping as indicator of independent social variables. A longitudinal study of former English pupils. (tesis de máster, University of Postdam).

Honey, J. (1989). Does Accent Matter? London: Faber and Faber.

Iannàccaro, G. y V. Dell'Aquila. (2001). "Mapping languages from inside: notes on perceptual dialectology". Social and Cultural Geography 2 (3), pp. 265-280.

Lope Blanch, J.M. (1986). El estudio del español hablado culto: historia de un proyecto, México D.F.: UNAM.

López García, Á. (1998). "Los conceptos de lengua y de dialecto a la luz de la teoría de prototipos". La Torre, I, pp. 7-19.

López Morales, H. (2004 [1989]). Sociolingüística. Madrid: Gredos (3º edición, muy corregida y aumentada).

López-Ornat, S. (1992). "Sobre la gramaticalización. Prototipos para la adquisición de la concordancia verbo-sujeto: datos de lengua española en niños de 1,6 a 3,6”. Cognitiva, 4 (1), pp. 48-74.

Martí Sánchez, M. (2004). "Bases para una gramática emergente y situada". Lingüistica en la Red (LINRED), 2, pp. 1-32.

Mase, Y. (1964). "Une nouvelle tentative pour tracer les frontières subjectives des dialectes". Orbis 13, pp. 357-379.

Menéndez Pidal, R. (1962). El dialecto leonés. Oviedo: Instituto de Estudios Asturianos, Diputación de Oviedo. [Incluye "Notas sobre el habla de Lena", publicado por primera vez en 1897 en la colección Asturias, y El dialecto leonés, publicado en 1906 en la Revista de Archivos, Bibliotecas y Museos].

Milroy, J. (2001). "Language ideologies and the consequences of standardization". Journal of Sociolinguistics, 5(4), pp. 530-555.

Milroy, L. (1982). "Social network and linguistic focusing”. En Romaine, S. (ed.). Sociolinguistic Variation in Speech Communities. London: Edward Arnold, pp. 141-152.

Moats, L. (2000). Speech to print: Language essentials for teachers. Baltimore: Brookes.

Montgomery, C. y J. Beal (2011). "Perceptual Dialectology". En Maguire, W. y A. McMahon (eds). Analysing Variation in English. Cambridge, NY: Cambridge University Press, pp. 121-148.

Moreno Fernández, F. (2000). Qué español enseñar. Madrid: Arco Libros.

Moreno Fernández, F. (2001). "Prototipos y modelos de lengua", Carabela. Modelos de uso de la lengua española, 50, pp. 5-20. 
Moreno Fernández, F. (2007). “Adquisición de segundas lenguas y Sociolingüística” Revista de Educación, 343, pp. 55-70.

Moreno Fernández, J. y F. Moreno Fernández (2002), "Madrid Perceptions of Regional Varieties in Spain”. En Long y D. Preston (eds.) Handbook of Perceptual Dialectology 2, pp. 295-320.

Morgenthaler García, L. (2008). Identidad y pluricentrismo lingüistico. Hablantes canarios frente a la estandarización. Frankfurt/Madrid: Vervuert Iberoamericana.

Neira Martínez, J. (1989). "Las fronteras del leonés". En Peira, P. et al. (eds.). Homenaje a Alonso Zamora Vicente II. Madrid: Castalia, pp. 215-225..

Palomino Hernández, M. C. (2013). Creencias, actitudes y motivación hacia el aprendizaje de ELE por parte de adolescentes sicilianos sin conocimientos previos de español. (memoria de máster). - Universidad de Jaén y Fundación Universitaria Iberoamericana.

Preston, D. (1989). Perceptual Dialectology. Nonliguists' Views of Areal Linguistics. DordrechtProvidence: Foris Pub.

Preston, D. (1993). “The Uses of Folk Linguistics". International Journal of Applied Linguistics, 3 (2), pp. 181-259.

Preston, D. (1999). Handbook of Perceptual Dialectology 1. Amsterdam: John Benjamins.

Preston, D. (2010). "Language, People, Salience, Space: Perceptual Dialectology and Language Regard”. Dialectologia, 5, pp. 87-131.

RAE (2009). Nueva gramática de la lengua española. Madrid: Espasa.

Rodríguez-Castellano, L. (1954). "Estado actual de la "H" aspirada en la provincia de Santander". Archivum 4, pp. 435-457.

Rojas, D. (2012). "Percepción y valoración de variedades geográficas del español de Chile entre hispanohablantes santiaguinos". Boletín de Filología, XLVII 1, pp. 137-163.

Trudgill, P. (1983a). Sociolinguistics: an Introduction to Language and Society. Harmondsworth; New York: Penguin.

Trudgill, P. (1983b). On Dialect: Social and Geographical Perspectives. Oxford: Blackwell.

Wells, J. (1982). Accents of English. Cambridge: CUP.

Zamora Vicente, A, (1979). Dialectología española. Madrid: Gredos.

Zistakis, A.H (2006). "Difference, $\sigma 0 \mu \pi \lambda$ ok$^{\prime}$ and the hierarchy of ideas in Plato's Sophist". Phronimon 7-2, pp. 29-45. 
